\title{
Selection of Apis mellifera L. Colonies for Quality Queen Rearing
}

\author{
Ajay Sharma*, Kiran Rana and Harish Kumar Sharma \\ Department of Entomology, Dr YS Parmar University of Horticulture and Forestry, \\ Nauni, Solan- 173230 H.P India. \\ *Corresponding author
}

\begin{tabular}{|c|c|}
\hline & A B S T R A C T \\
\hline $\begin{array}{l}\text { K e y w o r d s } \\
\text { Apis mellifera, } \\
\text { queen rearing, } \\
\text { colony strength, } \\
\text { brood area, } \\
\text { prolificness, honey } \\
\text { stores and pollen } \\
\text { stores }\end{array}$ & \multirow{3}{*}{ 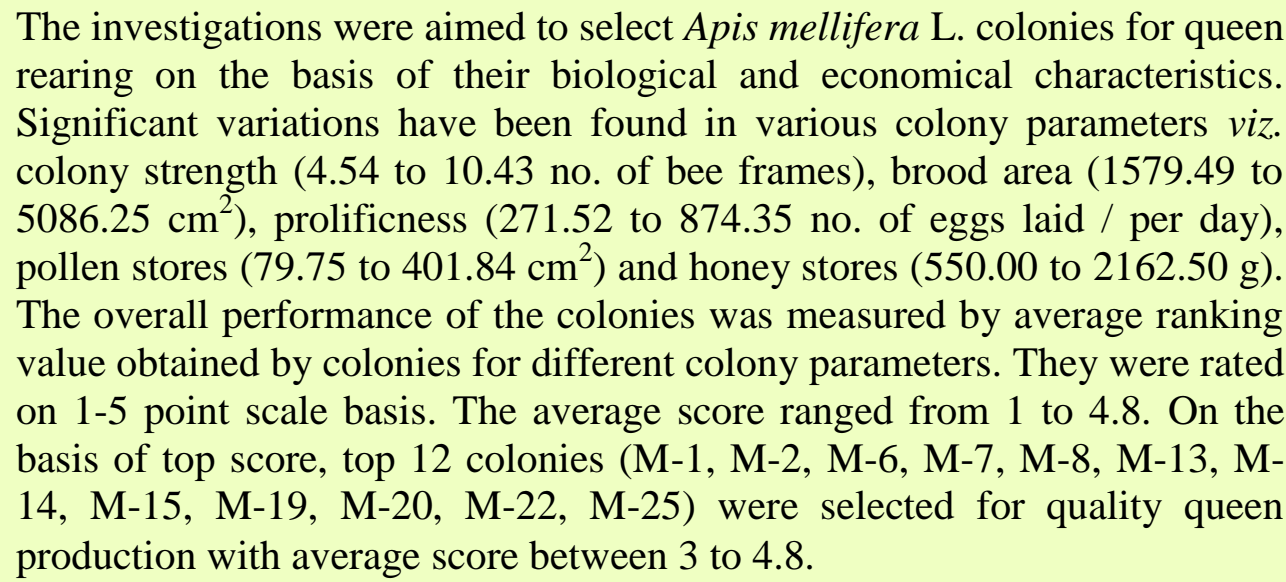 } \\
\hline Article I & \\
\hline $\begin{array}{l}\text { Accepted: } \\
\text { 22December } 2019 \\
\text { Available Online: } \\
\text { 20 January } 2020\end{array}$ & \\
\hline
\end{tabular}

\section{Introduction}

Honey bees are playing a crucial role in agriculture production systems. A good population of industrious workers of a honey bee colony is an important factor that contributes to higher build up and ultimately the high productivity of the colony and also good for pollination. This population is attained only through the best performance of queen. Colony strength and productivity of a stock of bees depend primarily on the age and qualities of its queen (Ruttner, 1988 and
Snelgrove, 1966). The European honey bee (Apis mellifera L.) is the basis of flourishing bee industry the world over. Since its introduction in India, a lot of need based research has been done on various aspects of management, bee flora, bee behavior, bee pathology. Bee breeding and stock improvement has remained neglected in India (Singh et al., 2007). There are possibilities of improvement of many characteristics of honey bee by means of selection of better bee stock. Therefore, studies on selection are greatly needed to identify honey bee colonies 
possessing desirable traits for further breeding programmes. A good quality queen can be reared from the better performing colonies so that the hereditary characters contributed by mother can be improved to a great extent, as the queen is the custodian of all the characteristics. Therefore, present studies on the selection of honey bee colonies were conducted for quality queen rearing.

\section{Materials and Methods}

The present investigations were carried out during March, 2018 to August, 2018, in apiary at the experimental farm of the Department of Entomology, College of Horticulture, Dr. YS Parmar University of Horticulture and Forestry, Nauni, Solan, Himachal Pradesh. The selection of honey bee colonies was based on biological and economic characteristics like colony strength, brood area, prolificness, honey stores, pollen stores etc. Twenty five A. mellifera L. colonies were taken randomly from the existing stock and the performance of these 25 A. mellifera L. colonies on following parameters was recorded at 21 days interval (Al-Tikrityet al., 1971) (Fig 1 and 2).

\section{Colony strength}

The strength of each experimental colony was estimated by counting the number of frames covered with brood and bees.

\section{Brood area}

The brood area in the experimental colonies was recorded with the help of 1 inch wire grid fixed on a brood frame which was later expressed in square centimetre by following formula (Chhuneja et al., 1992). The factor 6.45 was used to convert brood area from square inch to square centimetre.

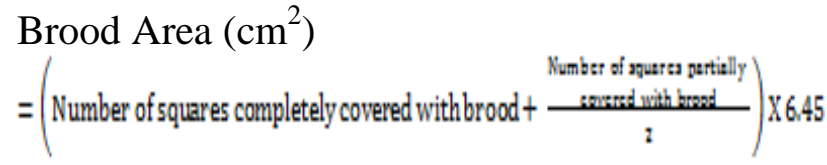

\section{Prolificness}

The egg laying rate of $A$. mellifera $\mathrm{L}$. queens of all 25 experimental colonies was calculated on the basis of total brood area.

Prolifieness $=\frac{\text { Total brood area }\left(\mathrm{cm}^{2}\right) \times 3.61}{21}$

The measured total brood area was multiplied by factor 3.61, which was the number of cells in one square centimetre of brood comb. The total number of brood cells was later divided by 21 to obtain the everyday egg laying rate of queen bee (Singh et al., 2007).

\section{Honey stores}

The amount of nectar stored was measured and expressed in grams by visually on the basis of assumption that one Langstroth frame sealed with honey weighed $2 \mathrm{~kg}$.

\section{Pollen stores}

The pollen stores of the colonies were measured by counting the number of cells in the wire grid covered by pollen. It is expressed in square centimetres.

\section{Rating of various performance characters}

The performance of experimental colonies of A. mellifera $\mathrm{L}$. was rated on the basis of 1-5 point scale. The ranking was done for the five different parameters i.e. colony strength, brood area, prolificness, pollen stores and honey stores. Ranking of least performing colony was given 1 point, whereas the best performing colony was ranked the highest rank 5. 


\section{Statistical Analysis}

The data recorded was significantly analyzed by using MS-Excel and OPSTAT. The mean value of data was subjected to statistical analysis as described by Gomez and Gomez (1986) by using Completely Randomized Design.

\section{Results and Discussion}

\section{Selection of A. mellifera $\mathrm{L}$. colonies}

Data on performance of 25 A. mellifera L. colonies (Table 1) revealed that the maximum (10.43 bee frames) average colony strength was recorded in colony number M-13 whereas the minimum in colony number $\mathrm{M}$ 18 (4.54 bee frames) which was statistically at par with colony number M-5 (4.96 bee frames) and M-16 (5.24 bee frames).

The maximum average brood area was recorded in colony number M-2 (5086.25 $\mathrm{cm}^{2}$ ). The minimum average brood area recorded in colony number M-16 (1579.49 $\mathrm{cm}^{2}$ ) was statistically at par to the average brood area of colony number M-18 (1785.89 $\left.\mathrm{cm}^{2}\right)$, M-12 (1961.21 $\left.\mathrm{cm}^{2}\right)$, M-5 (2031.69 $\left.\mathrm{cm}^{2}\right)$ and M-17 $\left(2539.18 \mathrm{~cm}^{2}\right)$.

The prolificness of the queen bee among the selected 25 colonies varied from 271.52 to 874.35 eggs laid /day. The maximum average prolificness was measured in colony number M-2 (874.35 eggs laid / day). The average prolificness was minimum and statistically similar in colony numbers M-16 (271.52), M18 (307.00), M-12 (337.14), M-5 (349.26) and M-17 (436.50). The data presented in Table 1 also revealed that the maximum average pollen stores were recorded in colony number M-2 $\left(401.84 \mathrm{~cm}^{2}\right)$ which was statistically at par to colony number M-6 $\left(336.46 \mathrm{~cm}^{2}\right)$ and the minimum average pollen stores $\left(79.75 \mathrm{~cm}^{2}\right)$ were recorded in colony number M-5 which was statistically similar to colony number $\mathrm{M}-12\left(81.38 \mathrm{~cm}^{2}\right)$, M-21 $\left(97.13 \mathrm{~cm}^{2}\right)$ and M-18 $\left(127.25 \mathrm{~cm}^{2}\right)$.

Among the 25 colonies, maximum average honey stores were recorded in colony M-7 $(2162.5 \mathrm{~g})$. The colony number M-16 (550 g) was recorded with minimum average honey stores which were statistically at par with colony number M-18 (650.00 g), M-12 (737.50 g) and M-21 (750 g).

In present selection study, significant variations have been found in various colony parameters like colony strength (4.54 to 10.43 bee frames), brood area (1579.49 to 5086.25 $\mathrm{cm}^{2}$ ), prolificness (271.52 to 874.35 eggs laid / day), pollen stores (79.75 to $401.84 \mathrm{~cm}^{2}$ ) and honey stores (550 to $2162.5 \mathrm{~g}$ ) in existing stock of 25 A. mellifera $\mathrm{L}$. colonies in university apiary maintained at Nauni, Solan.

The present findings are in conformity with Thakur (1994) who selected better honey producing breeder colonies on the basis of biological and economical characters. Monika (2017) reported significant difference among colony parameters i.e. pollen stores, colony strength, brood area, honey stores in Nauni conditions. The variations in colony parameters were also reported by Singh et al. (2007) and Sharma (2010).

\section{Rating of various performance characters for selection of $A$. mellifera $\mathrm{L}$. colonies}

The performance of experimental colonies of A. mellifera $\mathrm{L}$. was rated on the basis of 1-5 point scale (Table 2). 
Table.1 Performance of 25 A. mellifera L. colonies from existing stock at Nauni, Solan during March 2018 to August 2018

\begin{tabular}{|c|c|c|c|c|c|}
\hline \multirow{2}{*}{$\begin{array}{l}\text { Colony } \\
\text { No }\end{array}$} & \multicolumn{5}{|c|}{ Colony parameters } \\
\hline & $\begin{array}{l}\text { Average colony strength } \\
\text { (No. of frames with bees) }\end{array}$ & $\begin{array}{c}\text { Average } \\
\text { brood area } \\
\left(\mathrm{cm}^{2}\right)\end{array}$ & $\begin{array}{c}\text { Prolificness } \\
\text { (eggs laid / } \\
\text { day) }\end{array}$ & $\begin{array}{c}\text { Average pollen } \\
\text { store } \\
\left(\mathrm{cm}^{2}\right)\end{array}$ & $\begin{array}{l}\text { Average honey } \\
\text { stores } \\
\text { (g) }\end{array}$ \\
\hline M-1 & 8.96 & $\begin{array}{l}4113.00 \\
(3.61)^{*}\end{array}$ & $\begin{array}{l}707.04 \\
(2.84)\end{array}$ & $\begin{array}{l}227.50 \\
(2.34)\end{array}$ & $\begin{array}{c}1582.13 \\
(3.15)\end{array}$ \\
\hline M-2 & 9.94 & $\begin{array}{c}5086.25 \\
(3.71)\end{array}$ & $\begin{array}{l}874.35 \\
(2.94)\end{array}$ & $\begin{array}{l}401.84 \\
(2.56)\end{array}$ & $\begin{array}{c}1662.50 \\
(3.20)\end{array}$ \\
\hline M-3 & 8.04 & $\begin{array}{c}2808.43 \\
(3.45)\end{array}$ & $\begin{array}{c}482.78 \\
(2.68)\end{array}$ & $\begin{array}{l}151.38 \\
(2.16)\end{array}$ & $\begin{array}{c}1175.00 \\
(3.05)\end{array}$ \\
\hline M-4 & 6.79 & $\begin{array}{c}2574.11 \\
(3.41)\end{array}$ & $\begin{array}{c}442.50 \\
(2.64)\end{array}$ & $\begin{array}{l}201.70 \\
(2.30)\end{array}$ & $\begin{array}{l}987.50 \\
(2.99)\end{array}$ \\
\hline M-5 & 4.96 & $\begin{array}{c}2031.69 \\
(3.30)\end{array}$ & $\begin{array}{c}349.26 \\
(2.54)\end{array}$ & $\begin{array}{l}79.75 \\
(1.88)\end{array}$ & $\begin{array}{l}875.00 \\
(2.93)\end{array}$ \\
\hline M-6 & 9.71 & $\begin{array}{c}4761.76 \\
(3.68)\end{array}$ & $\begin{array}{l}818.57 \\
(2.91)\end{array}$ & $\begin{array}{c}336.46 \\
(2.51)\end{array}$ & $\begin{array}{c}1475.00 \\
(3.14)\end{array}$ \\
\hline M-7 & 10.06 & $\begin{array}{c}4895.08 \\
(3.69)\end{array}$ & $\begin{array}{l}841.49 \\
(2.92)\end{array}$ & $\begin{array}{l}224.26 \\
(2.33)\end{array}$ & $\begin{array}{c}2162.50 \\
(3.29)\end{array}$ \\
\hline M-8 & 9.39 & $\begin{array}{c}4596.02 \\
(3.66)\end{array}$ & $\begin{array}{l}790.08 \\
(2.90)\end{array}$ & $\begin{array}{l}201.54 \\
(2.29)\end{array}$ & $\begin{array}{c}1781.25 \\
(3.21)\end{array}$ \\
\hline M-9 & 7.74 & $\begin{array}{c}2994.86 \\
(3.48)\end{array}$ & $\begin{array}{c}514.83 \\
(2.71)\end{array}$ & $\begin{array}{l}150.38 \\
(2.16)\end{array}$ & $\begin{array}{c}1318.75 \\
(3.10)\end{array}$ \\
\hline M-10 & 7.85 & $\begin{array}{c}3031.54 \\
(3.48)\end{array}$ & $\begin{array}{l}521.14 \\
(2.72)\end{array}$ & $\begin{array}{l}140.50 \\
(2.14)\end{array}$ & $\begin{array}{c}950.00 \\
(2.95)\end{array}$ \\
\hline M-11 & 7.03 & $\begin{array}{c}2644.69 \\
(3.42)\end{array}$ & $\begin{array}{l}454.63 \\
(2.66)\end{array}$ & $\begin{array}{l}136.63 \\
(2.13)\end{array}$ & $\begin{array}{c}1037.50 \\
(2.99)\end{array}$ \\
\hline M-12 & 5.29 & $\begin{array}{c}1961.21 \\
(3.29)\end{array}$ & $\begin{array}{c}337.14 \\
(2.53)\end{array}$ & $\begin{array}{l}81.38 \\
(1.87)\end{array}$ & $\begin{array}{l}737.50 \\
(2.85)\end{array}$ \\
\hline M-13 & 10.43 & $\begin{array}{c}4001.95 \\
(3.60)\end{array}$ & $\begin{array}{c}687.95 \\
(2.83)\end{array}$ & $\begin{array}{l}252.39 \\
(2.39)\end{array}$ & $\begin{array}{c}1816.25 \\
(3.25)\end{array}$ \\
\hline M-14 & 9.96 & $\begin{array}{c}3741.97 \\
(3.57)\end{array}$ & $\begin{array}{c}643.26 \\
(2.80)\end{array}$ & $\begin{array}{l}245.54 \\
(2.38)\end{array}$ & $\begin{array}{c}1812.50 \\
(3.23)\end{array}$ \\
\hline M-15 & 8.38 & $\begin{array}{c}3005.45 \\
(3.47)\end{array}$ & $\begin{array}{l}516.65 \\
(2.71)\end{array}$ & $\begin{array}{l}203.51 \\
(2.28)\end{array}$ & $\begin{array}{c}1425.00 \\
(3.12)\end{array}$ \\
\hline M-16 & 5.24 & $\begin{array}{c}1579.49 \\
(3.19)\end{array}$ & $\begin{array}{c}271.52 \\
(2.43)\end{array}$ & $\begin{array}{l}88.38 \\
(1.91)\end{array}$ & $\begin{array}{l}550.00 \\
(2.70)\end{array}$ \\
\hline M-17 & 7.14 & $\begin{array}{c}2539.18 \\
(3.22)\end{array}$ & $\begin{array}{c}436.50 \\
(2.46)\end{array}$ & $\begin{array}{l}129.88 \\
(2.10)\end{array}$ & $\begin{array}{c}1075.00 \\
(3.02)\end{array}$ \\
\hline M-18 & 4.54 & $\begin{array}{c}1785.89 \\
(3.24)\end{array}$ & $\begin{array}{c}307.00 \\
(2.48)\end{array}$ & $\begin{array}{l}127.25 \\
(2.09)\end{array}$ & $\begin{array}{c}650.00 \\
(2.80)\end{array}$ \\
\hline M-19 & 8.83 & $\begin{array}{c}3501.03 \\
(3.54)\end{array}$ & $\begin{array}{c}601.84 \\
(2.78)\end{array}$ & $\begin{array}{l}152.56 \\
(2.17)\end{array}$ & $\begin{array}{c}1431.25 \\
(3.11)\end{array}$ \\
\hline M-20 & 9.89 & $\begin{array}{c}3389.65 \\
(3.53)\end{array}$ & $\begin{array}{c}582.70 \\
(2.76)\end{array}$ & $\begin{array}{l}182.64 \\
(2.26)\end{array}$ & $\begin{array}{c}1450.00 \\
(3.09)\end{array}$ \\
\hline M-21 & 5.95 & $\begin{array}{c}2252.01 \\
(3.35)\end{array}$ & $\begin{array}{c}387.13 \\
(2.59)\end{array}$ & $\begin{array}{l}97.13 \\
(1.98)\end{array}$ & $\begin{array}{l}750.00 \\
(2.86)\end{array}$ \\
\hline
\end{tabular}




\begin{tabular}{|c|c|c|c|c|c|}
\hline M-22 & 9.99 & 4031.38 & 693.01 & 210.60 & 1707.50 \\
& & $(3.61)$ & $(2.84)$ & $(2.28)$ & $(3.19)$ \\
\hline M-23 & 6.75 & 2798.33 & 481.05 & 135.00 & 1225.00 \\
& & $(3.44)$ & $(2.68)$ & $(2.11)$ & $(3.04)$ \\
\hline M-24 & 7.15 & 3228.56 & 555.00 & 201.58 & 1025.00 \\
& & $(3.50)$ & $(2.74)$ & $(2.30)$ & $(2.99)$ \\
\hline M-25 & 8.11 & 3410.27 & 586.24 & 173.63 & 1412.50 \\
& & $(3.53)$ & $(2.76)$ & $(2.23)$ & $(3.13)$ \\
\hline CD $_{(\mathbf{0 . 0 5})}$ & $\mathbf{0 . 7 3}$ & $\mathbf{0 . 1 5}$ & $\mathbf{0 . 1 5}$ & $\mathbf{0 . 1 5}$ & $\mathbf{0 . 1 7}$ \\
\hline
\end{tabular}

*Figures in parentheses are $\log (\mathrm{x}+1)$ transformed values

Table.2 Ranking of characters listed for selection of A. mellifera L.

\begin{tabular}{|c|c|c|}
\hline Characters & Rating & Range \\
\hline \multirow{5}{*}{$\begin{array}{c}\text { Colony Strength } \\
\text { (No. of frames with bees) }\end{array}$} & 1 & $4.54-5.72$ \\
\hline & 2 & $5.72-6.90$ \\
\hline & 3 & $6.90-8.08$ \\
\hline & 4 & $8.08-9.26$ \\
\hline & 5 & 9.26 and Above \\
\hline \multirow{5}{*}{$\begin{array}{l}\text { Brood area } \\
\quad\left(\mathrm{cm}^{2}\right)\end{array}$} & 1 & $1579.49-2280.84$ \\
\hline & 2 & $2280.84-2982.19$ \\
\hline & 3 & $2982.19-3683.54$ \\
\hline & 4 & $3683.54-4384.89$ \\
\hline & 5 & 4384.89 and Above \\
\hline \multirow{5}{*}{$\begin{array}{c}\text { Prolificness } \\
\text { (No of eggs laid / day) }\end{array}$} & 1 & $271.52-392.09$ \\
\hline & 2 & $392.09-512.66$ \\
\hline & 3 & $512.66-633.23$ \\
\hline & 4 & $633.23-753.80$ \\
\hline & 5 & 753.80 and Above \\
\hline \multirow{5}{*}{$\begin{array}{l}\text { Pollen stores } \\
\qquad\left(\mathrm{cm}^{2}\right)\end{array}$} & 1 & $88.38-144.17$ \\
\hline & 2 & $144.17-208.59$ \\
\hline & 3 & $208.59-273.01$ \\
\hline & 4 & $273.01-337.43$ \\
\hline & 5 & 337.43 and Above \\
\hline \multirow{5}{*}{$\begin{array}{c}\text { Honey stores } \\
\text { (g) }\end{array}$} & 1 & $550.00-872.50$ \\
\hline & 2 & $872.50-1195.00$ \\
\hline & 3 & $1195.00-1517.50$ \\
\hline & 4 & $1517.50-1840.00$ \\
\hline & 5 & 1840.00 and Above \\
\hline
\end{tabular}


Table.3 Ranking of $25 \mathrm{~A}$. mellifera colonies on 5 point scale for various performance characteristics

\begin{tabular}{|c|c|c|c|c|c|c|}
\hline \multirow[t]{2}{*}{ Colony number } & \multicolumn{6}{|c|}{ Scored values on 5 point scale } \\
\hline & $\begin{array}{c}\text { Average } \\
\text { colony } \\
\text { strength(no of } \\
\text { frames with } \\
\text { bees) }\end{array}$ & $\begin{array}{c}\text { Average } \\
\text { brood area } \\
\left(\mathrm{cm}^{2}\right)\end{array}$ & $\begin{array}{l}\text { Average } \\
\text { prolificness } \\
\text { (eggs laid / } \\
\text { day) }\end{array}$ & $\begin{array}{c}\text { Average } \\
\text { pollen stores } \\
\left(\mathrm{cm}^{2}\right)\end{array}$ & $\begin{array}{c}\text { Average } \\
\text { honey stores } \\
\text { (g) }\end{array}$ & Grand mean \\
\hline M-1 & 4 & 4 & 4 & 3 & 4 & 3.80 \\
\hline M-2 & 5 & 5 & 5 & 5 & 4 & 4.80 \\
\hline M-3 & 3 & 2 & 2 & 2 & 2 & 2.20 \\
\hline M-4 & 2 & 2 & 2 & 2 & 2 & 2.00 \\
\hline M-5 & 1 & 1 & 1 & 1 & 2 & 1.20 \\
\hline M-6 & 5 & 5 & 5 & 4 & 3 & 4.40 \\
\hline M-7 & 5 & 5 & 5 & 3 & 5 & 4.60 \\
\hline M-8 & 5 & 5 & 5 & 2 & 4 & 4.20 \\
\hline M-9 & 3 & 3 & 3 & 2 & 3 & 2.80 \\
\hline M-10 & 3 & 3 & 3 & 1 & 2 & 2.40 \\
\hline M-11 & 3 & 2 & 2 & 1 & 2 & 2.00 \\
\hline M-12 & 1 & 1 & 1 & 1 & 1 & 1.00 \\
\hline M-13 & 5 & 4 & 4 & 3 & 4 & 4.00 \\
\hline M-14 & 5 & 4 & 4 & 3 & 4 & 4.00 \\
\hline M-15 & 4 & 3 & 3 & 2 & 3 & 3.00 \\
\hline M-16 & 1 & 1 & 1 & 1 & 1 & 1.00 \\
\hline M-17 & 3 & 2 & 2 & 1 & 2 & 2.00 \\
\hline M-18 & 1 & 1 & 1 & 1 & 1 & 1.00 \\
\hline M-19 & 4 & 3 & 3 & 2 & 3 & 3.00 \\
\hline M-20 & 5 & 3 & 3 & 2 & 3 & 3.20 \\
\hline M-21 & 2 & 1 & 1 & 1 & 1 & 1.20 \\
\hline M-22 & 5 & 4 & 4 & 3 & 4 & 4.00 \\
\hline M-23 & 2 & 2 & 2 & 1 & 3 & 2.00 \\
\hline M-24 & 3 & 3 & 3 & 2 & 2 & 2.60 \\
\hline M-25 & 4 & 3 & 3 & 2 & 3 & 3.00 \\
\hline
\end{tabular}




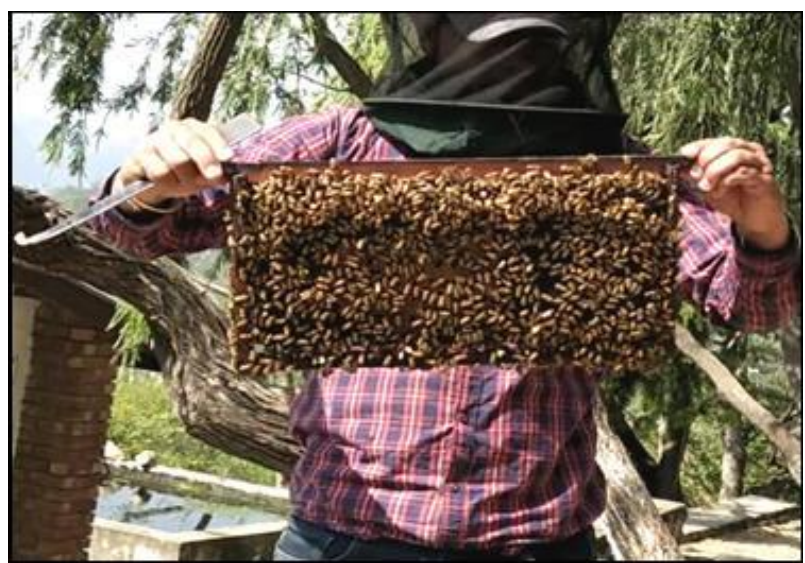

Figure.1 Visual observation of hive

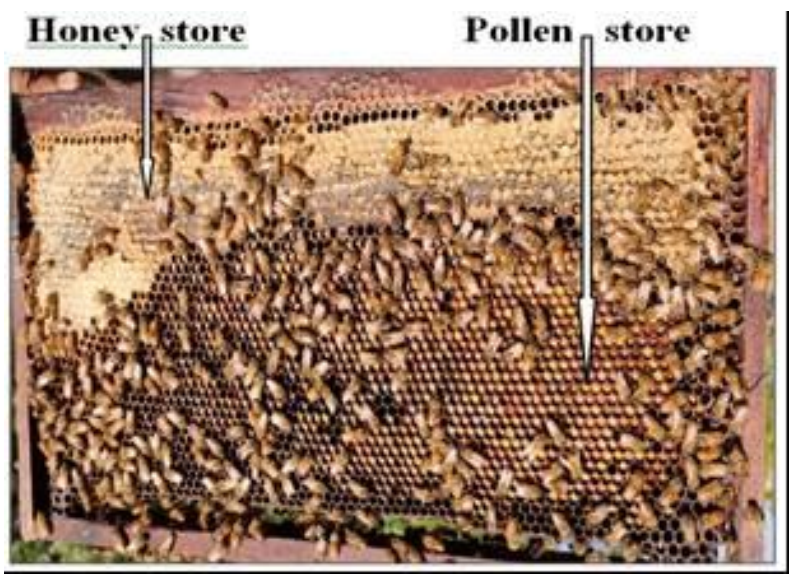

Figure.2 Bee frame

\section{Ranking of 25 A. mellifera $\mathrm{L}$. colonies for performance characteristics}

The scoring of 25 A. mellifera colonies on the basis of their performance has been presented in Table 3. The overall performance of a colony was measured by average ranking value of the colony strength, brood area, prolificness, pollen stores and honey stores. The average score ranged from 1 to 4.8. On the basis of top score, 12 colonies (M-1, M-2, M-6, M-7, M-8, M-13, M-14, M-15, M-19, M-20, M-22, M-25) were selected for queen rearing having average score ranged between 3 to 4.8 .

In present study ranking and rating of honey bee colonies have been done on the basis of 5 point scoring system for each of the attributes and top score twelve colonies were selected for queen rearing. Singh et al. (2007) also used five-point scale for scoring of various performance characters to select better performing A. mellifera colonies for stock improvement programmed. Similarly, $A$. cerana (Anonymous, 2010) and A. mellifera (Sharma et al., 2017) colonies were ranked on five point scale and selected for further studies.

Significant variations have been found in various colony parameters like colony strength, brood area, prolificness, pollen stores and honey stores in the existing stock of 25 colonies in University apiary maintained at Nauni, Solan. These variations allow beekeepers and researchers to select best performing colonies from existing stock.

\section{Acknowledgement}

The authors are highly thankful to Department of Entomology, Dr. YS Parmar University of Horticulture and Forestry, Nauni, Solan-173230 (Himachal Pradesh) INDIA.

\section{References}

Al-Tikrity WS, Hillman RC, Benton AW and Clarke WWJ. 1971. New instrument for brood measurement in a honey bee colony. American Bee Journal 111: 2021.

Anonymous. 2010. Annual Report: All India Coordinated project on honey bees and pollinators. 44p.

Chhuneja PK, Brar HS and Goyal NP. 1992. Studies on some pollen substitutes fed as moist patty to Apis mellifera L. colonies. II. Effect on colony development. Indian Bee Journal 55: 17-25.

Gomez KA and Gomez AA. 1986. Statistical 
procedures for agricultural research. John Wiley and Sons, New York. 680p. Monika. 2017. Studies on apple pollen foraging preference in Apis mellifera $\mathrm{L}$. M.Sc. Thesis. Dr. YS Parmar University of Horticulture and Forestry, Nauni, Solan. 49p.

Ruttner F. 1988. Biogeography and Taxonomy of Honey bees. Springer Verlag Heidelberg, Berlin, New York. $284 \mathrm{p}$.

Sharma R. 2010. Screening of Apis mellifera L. colonies for Varroa tolerance and evalustion of colony perforance of selected stock. Ph.D. Thesis. Dr YS Parmar University of Horticulture and Forestry, Nauni, Solan. 145p.

Sharma HK, Monika, Rana K and Thakur M. 2017.Selected high and low pollen hoarding colonies for Apis mellifera for apple foraging preference. International Journal of Current Microbiology and Applied Sciences 6: 2233-44.

Singh B, Gatoria GS and Chhuneja PK. 2007.Selection of best performing Apis mellifera colonies for stock improvement programme. Indian Bee Journal 69: 1-7.

Snelgrove LE (ed). 1966. Queen Rearing. $3^{\text {rd }}$ ed. SnelgroveBleadon, Somerset, Landon. 344p.

Thakur RK. 1994. Studies on the breeding of honey bees Apis mellifera L. for honey production through artificial insemination. Ph.D Thesis.Dr. YS Parmar University of Horticulture and Forestry, Nauni, Solan. 155p.

\section{How to cite this article:}

Ajay Sharma, Kiran Rana and Harish Kumar Sharma. 2020. Selection of Apis mellifera L. Colonies for Quality Queen Rearing. Int.J.Curr.Microbiol.App.Sci. 9(01): 2407-2414. doi: https://doi.org/10.20546/ijcmas.2020.901.274 